\title{
First-Principles Determination of the Ground-State Structure of $\mathrm{LiBH}_{4}$
}

\author{
Adem Tekin, ${ }^{1, *}$ Riccarda Caputo, ${ }^{2}$ and Andreas Züttel ${ }^{2}$ \\ ${ }^{1}$ Informatics Institute, Istanbul Technical University, 34469 Maslak, Istanbul, Turkey \\ ${ }^{2}$ EMPA, Swiss Federal Laboratories for Materials Science and Technology, Überlandstrasse, 129 CH-8600 Dübendorf, Switzerland
}

(Received 15 December 2009; published 24 May 2010)

\begin{abstract}
The potential energy surface of $\mathrm{LiBH}_{4}$ is investigated by a ground-state search method based on simulated annealing and first-principles density functional theory calculations. A new stable orthogonal structure with Pnma symmetry is found, which is $9.66 \mathrm{~kJ} / \mathrm{mol}$ lower in energy than the proposed Pnma structure by Soulié et al. [J. Alloys Compd. 346, 200 (2002)]. For the high-temperature structure, we suggest a new monoclinic $P 2 / c$ structure, which is $21.26 \mathrm{~kJ} / \mathrm{mol}$ over the ground-state energy and shows no lattice instability.
\end{abstract}

DOI: 10.1103/PhysRevLett.104.215501

PACS numbers: 61.66.Fn, 71.15.Mb, 71.15.Nc

Lithium borohydride $\left(\mathrm{LiBH}_{4}\right)$ has been extensively studied since the early 1940s, when Schlesinger and coworkers started a systematic synthesis of metal borohydrides [1]. Although a considerable number of papers have been published on $\mathrm{LiBH}_{4}$, a clear theoretical structure determination from first principles seems to suffer from a lack of methodological approach. On the experimental side, already Harris et al. [2] reported an orthorhombic structure (space group Pcmn) with cell dimensions $a=6.81 \AA$, $b=4.43 \AA$, and $c=7.17 \AA$. In the last decade, because $\mathrm{LiBH}_{4}$ has been proposed [3] as a promising material for technological applications of hydrogen as clean fuel, thanks to its high gravimetric $(18.5 \mathrm{wt} \% \mathrm{H})$ and volumetric $\left(121 \mathrm{~kg} \mathrm{H} / \mathrm{m}^{3}\right)$ hydrogen density, the interest in the entire class of metal tetrahydroborohydrides has been rewound. Soulié et al. [4] reported an orthorhombic structure [space group Pnma (IT 62), $a=7.17858 \AA, b=4.43686 \AA$, and $c=6.80321 \AA]$ by synchrotron x-ray diffraction at room temperature. In this structure, each $\left[\mathrm{BH}_{4}\right]^{\delta-}$ anion was surrounded by four $\mathrm{Li}^{\delta+}$ cations and each $\mathrm{Li}^{\delta+}$ by four $\left[\mathrm{BH}_{4}\right]^{\delta-}$ both in tetrahedral configuration. The $\left[\mathrm{BH}_{4}\right]^{\delta-}$ ions were strongly distorted from the ideal tetrahedral geometry. The same Pnma structure was also reported by Züttel et al. [5]. Furthermore, Soulié et al. [4] observed that Pnma structure transforms into a hexagonal phase (space group $P 6_{3} m c, \quad a=4.27631 \AA$, and $c=$ $6.94844 \AA$ ) at $408 \mathrm{~K}$. However, this structure was found to have imaginary normal modes of vibration in the theoretical calculations performed a few years later [6] and therein a monoclinic structure with symmetry group $C c$ was proposed for the high-temperature phase of $\mathrm{LiBH}_{4}$. Recently, a single crystal synchrotron diffraction study was conducted by Filinchuk et al. [7] aiming to find the more accurate low- and high-temperature crystal structures of $\mathrm{LiBH}_{4}$. Correspondingly, they [7] confirmed the Pnma with a nearly ideal tetrahedral $\left[\mathrm{BH}_{4}\right]^{\delta-}$ ions for the low temperature structure and the $P 6_{3} m c$ structure for the hightemperature phase. In contrast to Soulié et al. [4], they predicted the phase transition occurring at $381 \mathrm{~K}$.
Moreover, at room temperature, a new phase with Ama2 symmetry [8] has been reported by an x-ray diffraction study. It has also been found that pressure-induced phase transition is possible in $\mathrm{LiBH}_{4}$ : at room temperature and a pressure of 1.2-10 GPa a transition from Pnma to Ama2 phase occurs and above $10 \mathrm{GPa}$ a cubic $(F m \overline{3} m)$ $\mathrm{NaBH}_{4}$-type structure is preferred. In Ama2 structure, $\mathrm{Li}^{\delta+}$ ions prefer a tetrahedral coordination with the $\left[\mathrm{BH}_{4}\right]^{\delta-}$ anions and each $\left[\mathrm{BH}_{4}\right]^{\delta-}$ group is surrounded by a square-planar coordination of four $\mathrm{Li}^{\delta+}$ atoms. Differently, in $F m \overline{3} m$ structure, each of the $\mathrm{Li}^{\delta+}$ and $\left[\mathrm{BH}_{4}\right]^{\delta-}$ ions are octahedrally coordinated. It is clear that despite the enormous efforts and the large amount of work devoted to the study of such an interesting compound, a clear determination of the ground-state structure has not yet been unambiguously defined. We report in the present Letter the first-principles structure determination of $\mathrm{LiBH}_{4}$, following the same procedure already applied for the ground-state determination of $\mathrm{Mg}\left(\mathrm{BH}_{4}\right)_{2}$ [9] and $\mathrm{Mg}\left(\mathrm{NH}_{3}\right)_{n} \mathrm{Cl}_{2}$ [10] with $n=6,2,1$.

As discussed in [9] for $\mathrm{Mg}\left(\mathrm{BH}_{4}\right)_{2}$, the number of bonds between the metal atom and hydrogen stabilizes the crystal structure. The crystal structures of $\mathrm{LiBH}_{4}$ were constructed by maximizing the number of $\mathrm{Li}-\mathrm{H}$ bonds within a $2 \times$ $2 \times 2$ supercell, using only several bond length constraints, and 1,2, and 4 formula units (f.u.) of $\mathrm{LiBH}_{4}$. A model with 2 f.u. of $\mathrm{LiBH}_{4}$ used in simulated annealing (SA) search [11] is shown in Fig. 1, where (i) one of the Li atom was set to the origin, (ii) a fixed tetrahedral coordinate system was used for $\left[\mathrm{BH}_{4}\right]^{\delta-}$ with $1.24 \AA \mathrm{B}-\mathrm{H}$ distances, (iii) the positions of the $\mathrm{Li}$ atom and $\left[\mathrm{BH}_{4}\right]^{\delta-}$ groups were determined using three spherical coordinates (the center of mass distance, $\Theta$ and $\Phi)$, (iv) three Euler angle parameters ( $\alpha$, $\beta$, and $\gamma$ ) were used to rotate each $\left[\mathrm{BH}_{4}\right]^{\delta-}$ group, (v) the lattice vectors were used as parameters, and (vi) the resulting 24 parameters (15 and 42 for 1 and 4 f.u., respectively) were globally optimized to maximize the number of $\mathrm{Li}-\mathrm{H}$ bonds in $\mathrm{LiBH}_{4}$. In addition to this model, in the SA search, the type of crystal system, e.g., triclinic and mono- 


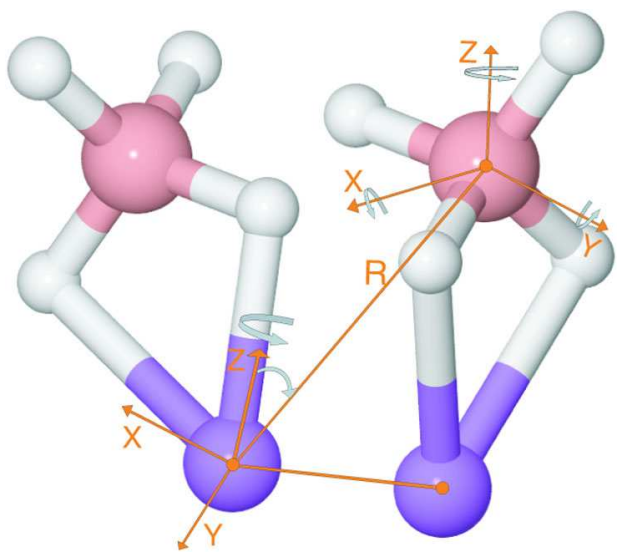

FIG. 1 (color online). The model system used in SA optimizations. (See details in the text.)

clinic, was also applied as a constraint to be able to reduce the parameter search space. Since our SA optimization approach is based only on the geometrical features of the studied system [neither empirical potentials nor density functional theory (DFT) energies are used], bond length constraints must be carefully introduced into the models discussed above to avoid results leading to unphysical crystal structure. More specifically, we adopted the cutoff criterion that if the $\mathrm{Li}-\mathrm{B}, \mathrm{Li}-\mathrm{Li}, \mathrm{B}-\mathrm{B}$, and $\mathrm{H}-\mathrm{H}$ distances in the $2 \times 2 \times 2$ supercell were longer than $2.55,3.75,3.78$, and $2.3 \AA$, respectively, then the corresponding crystal structures were accepted in these model systems. The fitness criteria in the SA optimizations, namely, the total number of $\mathrm{Li}-\mathrm{H}$ bonds in the cutthrough lattice, were determined by simply counting the number of $\mathrm{Li}-\mathrm{H}$ bonds, for the $\mathrm{Li}-\mathrm{H}$ bond in the ranges $(2.00,2.50) \AA$ or $(1.84,2.50) \AA$. All of these constraints were adjusted with the help of data reported in the literature. The best promising structures were further optimized by the subsequent periodic lattice calculations.

We employed DFT based methods, in particular, CASTEP [12], as implemented in MATERIALS STUDIO 4.4 to optimize ionic positions and lattice parameters of $\alpha$-boron [13], lithium (bcc), and all the structures reported in the present Letter. Norm-conserving pseudopotentials were utilized for all atoms together with a fine mesh of $k$ points, with the energy conversion threshold of $0.01 \mathrm{meV} /$ atom, maximum displacement of $0.001 \AA$ and maximum force of $0.03 \mathrm{eV} / \AA$, yielding a high accuracy for the energy and atomic displacements. For lithium and boron atoms the valence region was modeled using the $2 s^{1}$ and $2 s^{2} 2 p^{1}$ electrons, respectively. The Perdew-Burke-Ernzerhof 96 and the generalized gradient form (GGA-PBE) of the exchange-correlation functional were applied. Density functional perturbation theory (DFPT) as implemented in CASTEP [14] was used for phonon calculations. In addition, the fully optimized structures calculated by CASTEP were further considered in the phonon calculations performed by plane-wave self-consistent field (PWSCF) which is a computer code for electronic-structure calculations within DFT and density functional perturbation theory, using pseudopotentials and a plane-wave basis set [15].

The SA search successfully predicted many distinct structures for $\mathrm{LiBH}_{4}$, with different molecular configurations, e.g., bidentate and tridentate, 25 of them were selected for the periodic DFT calculations. In addition to those structures, we considered the two most important experimentally determined structures, namely, orthorhombic and hexagonal $[3,4,16]$ (and references therein), in the periodic lattice calculations. In Table I, we reported the structure data and thermodynamic results of selected structures whose lattice stability was confirmed by the phonon

TABLE I. Thermodynamic and structural data of selected structures, which were fully optimized by using CASTEP. The quantities reported are the enthalpy of formation $(\mathrm{kJ} / \mathrm{mol})$ at $T=0 \mathrm{~K}, \Delta_{f} H$, the symmetry group that best fitted the optimized structures, within brackets the international crystallographic number (IT), the corresponding lattice parameters ( $\AA$ ) and in brackets the maximum deviation of the atomic coordinates from the crystallographic positions corresponding to the specific group of symmetry. The first seven structures showed all the normal modes of vibration real. The last three structures were optimized starting from the reference structures, of which only the orthorhombic structure showed lattice stability. The cubic structure $F \overline{4} 3 m$, which was modeled in comparison to the cubic $\mathrm{NaBH}_{4}$ structure [17], showed imaginary modes.

\begin{tabular}{cccc}
\hline \hline$\Delta_{f} H$ & Symmetry group (IT) & Lattice parameters (max deviation) & Remarks \\
\hline-239.988 & $P n m a(62)$ & $8.484,4.348,5.750(0.02)$ & Orthorhombic \\
-218.724 & $P 2 / c(13)$ & $11.343,6.993,8.780(0.004)$ & Monoclinic \\
-234.854 & $C 2(5)$ & $10.550,10.016,6.732(0.004)$ & Monoclinic \\
-233.122 & $R 3(146)$ & $9.90,9.90,11.28(0.02)$ & Hexagonal \\
-234.516 & $C c(9)$ & $13.13,6.08,6.93(0.03)$ & Monoclinic \\
-235.079 & $P 2_{1} / c(14)$ & $4.15,7.01,7.81(0.02)$ & Monoclinic \\
-233.248 & $P 2_{1} 3(198)$ & $6.22,6.22,6.22(0.02)$ & Cubic \\
-188.976 & $F 43 m(216)$ & $5.741,5.741,5.741(0,01)$ & Oubic \\
-230.330 & $P n m a(62)$ & $7.248,4.367,6.559(0.001)$ & Hexagononombic [4] \\
-212.511 & $P 6_{3} m c(186)$ & $4.221,4.221,7.612(0.001)$ & $C$-centered orthorhombic [8] \\
-218.889 & $A m a 2(40)$ & $6.293,5.626,5.901(0.001)$ & \\
\hline \hline
\end{tabular}


analysis. As shown in Table I, we found six structures with different symmetry groups and enthalpy of formation in the range up $10 \mathrm{~kJ} / \mathrm{mol}$ lower than the orthorhombic structure of Soulié et al. [4]. The enthalpy of formation shown in Table I was calculated as a difference between the enthalpy of $\mathrm{LiBH}_{4}$ (with its specific phase) and the corresponding enthalpies of $\mathrm{Li}(\mathrm{bcc}), \alpha-\mathrm{B}$ [13], and $\mathrm{H}_{2}$ (calculated as an isolated molecule in a large cubic lattice).

The lowest energy structure, which is the first row in Table I, is the new orthorhombic structure (ionic coordinates are given in Table II) with Pnma symmetry. In that structure, each $\left[\mathrm{BH}_{4}\right]^{\delta-}$ group is coordinated by four $\mathrm{Li}^{\delta+}$ ions and each $\mathrm{Li}^{\delta+}$ ion by four $\left[\mathrm{BH}_{4}\right]^{\delta-}$ groups, both in distorted tetrahedral arrangements. In particular, $\mathrm{B}-\mathrm{Li}$ distances are 2.41-2.56 $\mathrm{A}, \mathrm{Li}-\mathrm{H}$ distances are 1.91$2.23 \AA$, Li- $\mathrm{Li}$ distances are 3.40-4.66 $\mathrm{A}$, and $\mathrm{B}-\mathrm{B}$ distances are 3.62-4.39 $\AA$. Furthermore, the $\left[\mathrm{BH}_{4}\right]^{\delta-}$ group forms an almost undistorted tetrahedron with equal $\mathrm{B}-\mathrm{H}$ distances $(1.22 \AA)$ and $\mathrm{H}-\mathrm{B}-\mathrm{H}$ angles $\left(108.64^{\circ}-\right.$ $\left.112.96^{\circ}\right)$. These values are in agreement with the neutron diffraction data at $3.5 \mathrm{~K}$ [16]. Clearly, the lithium-boron distances reflected a compromise between the Coulomb interaction of $\mathrm{Li}^{\delta+}$ cations with $\left[\mathrm{BH}_{4}\right]^{\delta-}$ anions and the correlation between occupied orbitals, extended over the borohydride group and the empty orbitals on lithium cation. In addition, since the hydrogen atoms on each $\left[\mathrm{BH}_{4}\right]^{\delta-}$ group were arranged tetrahedrally to boron and in a bidentate way to lithium, the latter atom was led to occupy the center of a tetrahedron formed by four $\left[\mathrm{BH}_{4}\right]^{\delta-}$ groups. The $\mathrm{Li}-\mathrm{B}$ distances in our case were larger than the reference orthorhombic structure [4] and very similar to the values reported by Harry et al. [2].

The most striking difference between the new orthorhombic structure and that reported by Soulié et al. [4] was the preference of number of $\mathrm{Li}-\mathrm{H}$ bonding, as shown in Fig. 2. More specifically, the former had only bidentate configurations between $\mathrm{Li}-\mathrm{H}$ whereas the latter had a mixture of bidentate and tridentate configurations: the hydrogen atoms of $\frac{1}{4}$ of the $\left[\mathrm{BH}_{4}\right]^{\delta-}$ group coordinated to $\mathrm{Li}$ tridentately and the remaining hydrogen atoms of $\frac{3}{4}$ of $\left[\mathrm{BH}_{4}\right]^{\delta-}$ groups bidentately. The differences in the local coordination of lithium were captured in the total electron density of states. While in the ground-state structure the distribution of the electron density of states was more homogeneous, in the Pnma structure of Soulié et al. [4]

TABLE II. DFT coordinates of the new orthorhombic structure of $\mathrm{LiBH}_{4}$.

\begin{tabular}{lcccr}
\hline \hline Ion type & Wyckoff position & $x$ & $y$ & \multicolumn{1}{c}{$z$} \\
\hline H1 & $8 d$ & 0.78275 & 0.47829 & 0.18183 \\
Li2 & $4 c$ & 0.35630 & 0.75000 & -0.41874 \\
B3 & $4 c$ & 0.86388 & 0.25000 & 0.15149 \\
H4 & $4 c$ & 0.97034 & 0.25000 & 0.29306 \\
H5 & $4 c$ & 0.91091 & 0.25000 & -0.04903 \\
\hline \hline
\end{tabular}

it showed a presence of differentiated states both in the $s$ region and the $p$ region [18]. In addition, the band gap was lower in our structure than in the reference structure: 6.225 and $6.871 \mathrm{eV}$, respectively. Moreover, the lattice parameters of the ground-state structure differed from those of Pnma structure [4]. Especially, $\vec{a}$ direction was enlarged and $\vec{c}$ direction was shortened in our Pnma structure. These cell parameters resulted in a slightly larger unit cell volume than the reference structure by only $4.5 \AA^{3}$

Actually our full geometry optimization of both the orthorhombic and hexagonal structures reported by Soulié et al. [4] gave different lattice parameters: $a$ increased by $0.95 \%$ and $c$ decreased by $3.59 \%$ in the orthorhombic structure and the $c$ parameter increased by $9.56 \%$ in the hexagonal structure compared to the corresponding reported value [4]. In between those two extrema, we found five different local minimum structures. Two almost isoenergetic structures, the $C 2$ and $C c$, only differed by $0.338 \mathrm{~kJ} / \mathrm{mol}$, while the structure with the hexagonal setting of the trigonal crystal system $R 3$ and the cubic $P 2{ }_{1} 3$ structures differed by $0.126 \mathrm{~kJ} / \mathrm{mol}$. The other orthorhombic structure $P 2_{1} / c$ resulted in $4.909 \mathrm{~kJ} / \mathrm{mol}$ above the

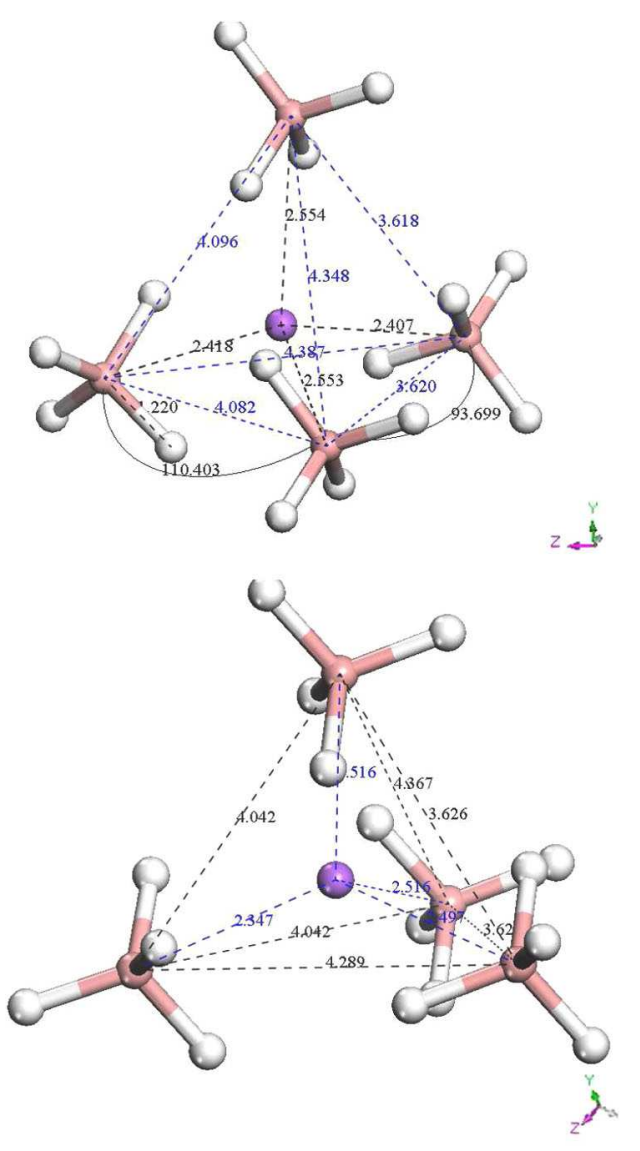

FIG. 2 (color online). (Top) The lowest energy structure of $\mathrm{LiBH}_{4}$. (Below) Fully optimized Pnma structure of Soulié et al. [4]. 
TABLE III. DFT coordinates of the high-temperature $P 2 / c$ structure of $\mathrm{LiBH}_{4}$.

\begin{tabular}{ccccr}
\hline \hline Ion type & Wyckoff position & $x$ & $y$ & \multicolumn{1}{c}{$z$} \\
\hline Li1 & $4 g$ & 0.1305 & 0.4976 & 0.7484 \\
B2 & $4 g$ & 0.8075 & 0.5522 & 0.0622 \\
H3 & $4 g$ & 0.9254 & 0.6570 & -0.0010 \\
H4 & $4 g$ & 0.8718 & 0.3988 & 0.9578 \\
H5 & $4 g$ & 0.6938 & 0.5165 & 0.2688 \\
H6 & $4 g$ & 0.7339 & 0.6374 & 0.0310 \\
\hline \hline
\end{tabular}

ground-state orthorhombic structure. The $C c$ symmetry structure [6], proposed as a candidate for the hightemperature phase, found $5.74 \mathrm{~kJ} / \mathrm{mol}$ higher than the ground-state structure and instable showing the first two normal modes with imaginary frequencies, $-70.43 \mathrm{~cm}^{-1}$ and $-10.97 \mathrm{~cm}^{-1}$. Conversely, one of our stable and higher energy structure, the monoclinic structure with symmetry $P 2 / c$, resulted in $21.264 \mathrm{~kJ} / \mathrm{mol}$ above the ground-state structure, and showed lattice stability by phonon analysis with all real normal modes [18]. The relaxed DFT ionic coordinates of this structure are given in Table III. Furthermore, we modeled a face-centered cubic (fcc) structure with $F \overline{4} 3 m$ symmetry, in which $\mathrm{Li}^{\delta+}$ and $\left[\mathrm{BH}_{4}\right]^{\delta-}$ ions are octahedrally coordinated, in analogy to $\mathrm{NaBH}_{4}$ [17]. Compared to the quasicubic structure with $R 3$ symmetry, the unitcell volume of the fcc structure was quite compact: $319.100 \AA^{3}$ versus $189.214 \AA^{3}$. As reported in Table I, the hexa-coordination of $\mathrm{Li}$ to borohydride groups in the $F \overline{4} 3 m$ symmetry was energetically unfavoured, being $51.012 \mathrm{~kJ} / \mathrm{mol}$ above the ground-state structure. In addition, the phonon analysis revealed a lattice instability, showing clearly that the hexa-coordination of $\mathrm{Li}^{\delta+}$ to $\left[\mathrm{BH}_{4}\right]^{\delta-}$ groups was dynamically unfavored. Compared to the analogous structure of $\mathrm{NaBH}_{4}$, the $\mathrm{LiBH}_{4}$ - fcc showed a shorter cubic lattice parameter and hence a reduced molar volume by $20.56 \%$ than of $\mathrm{NaBH}_{4}$, a fact that can be attributed mainly to steric effects, being the ionic radius of $\mathrm{Li}$ smaller than of $\mathrm{Na}$. Clearly, what differentiated the structures and governed both the lattice symmetry and the lattice stability was the local coordination of lithium atoms to $\left[\mathrm{BH}_{4}\right]^{\delta-}$ groups.

In conclusion, the potential energy surface of $\mathrm{LiBH}_{4}$ was investigated by a ground-state search method based on SA and first-principles DFT calculations. Six structures were found lower in energy than the reported orthorhombic structure. Even if the symmetry group of the lowest energy structure was the same, Pnma, the lattice parameters were different, leading to a slight difference in the cell volume [18]. For the high energy structure, we suggested a new monoclinic $P 2 / c$ over the hexagonal $P 6_{3} m c$, because of the lattice stability, being all the phonon frequencies posi- tive for the former, while the latter showed two double degenerate imaginary modes, with frequencies -55.70 and $-41.44 \mathrm{~cm}^{-1}$. Based on the hypothesis that a direct transition from the orthorhombic ground state to the monoclinic structure occurred, the transition temperature was estimated to be $408.8 \mathrm{~K}$. The first-principles structure determination approach that we used in the present work revealed a powerful tool for modeling and predicting stable structures.

*adem.tekin@be.itu.edu.tr

[1] H. I. Schlesinger and H. C. Brown, J. Am. Chem. Soc. 62, 3429 (1940).

[2] P. M. Harris and E. P. Meibohm, J. Am. Chem. Soc. 69, 1231 (1947).

[3] A. Züttel, P. Wenger, S. Rentsch, P. Sudan, Ph. Mauron, and Ch. Emmenegger, J. Power Sources 118, 1 (2003).

[4] J-Ph. Soulie', G. Renaudin, R. Cerny, and K. Yvon, J. Alloys Compd. 346, 200 (2002).

[5] A. Züttel, S. Rentsch, P. Fischer, P. Wenger, P. Sudan, Ph. Mauron, and Ch. Emmenegger, J. Alloys Compd. 356357, 515 (2003).

[6] Z. Łodziana and T. Vegge, Phys. Rev. Lett. 93, 145501 (2004).

[7] Y. Filinchuk, D. Chernyshov, and R. Cerny, J. Phys. Chem. C 112, 10579 (2008).

[8] Y. Filinchuk, D. Chernyshov, A. Nevidomskyy, and V. Dmitriev, Angew. Chem., Int. Ed. 47, 529 (2008).

[9] R. Caputo, A. Tekin, W. Sikora, and A. Züttel, Chem. Phys. Lett. 480, 203 (2009).

[10] A. Tekin, J.S. Hummelshøj, H.S. Jacobsen, D. Sveinbjörnsson, D. Blanchard, J. K. Nørskov, and T. Vegge, Energ. Environ. Sci. 3, 448 (2010).

[11] A. Corona, M. Marchesi, C. Martini, and S. Ridella, ACM Trans. Math. Softw. 13, 262 (1987).

[12] S. J. Clark, M. D. Segall, C. J. Pickard, P. J. Haspin, M. J. Probert, K. Refson, and M. C. Payne, Z. Kristallogr. 220, 567 (2005).

[13] R. Caputo and A. Züttel, Mol. Phys. 107, 1831 (2009).

[14] K. Refson, S. J. Clark, and P. R. Tulip, Phys. Rev. B 73, 155114 (2006).

[15] P. Giannozzi et al., J. Phys. Condens. Matter 21, 395502 (2009).

[16] M. R. Hartman, J. J. Rush, T. J. Udovic, R. C. Bowman, Jr., and S. J. Hwang, J. Solid State Chem. 180, 1298 (2007).

[17] P. Martelli, R. Caputo, A. Remhof, P. Mauron, A. Borgschulte, and A. Züttel, J. Phys. Chem. C 114, 7173 (2010).

[18] See supplementary material at http://link.aps.org/ supplemental/10.1103/PhysRevLett.104.215501 for the figures containing simulated x-ray diffraction patterns, density of states, electron density distributions, and high-temperature structure. 